\title{
Mejores prácticas empresariales de responsabilidad social en la inclusión de personas con discapacidad. Estudio de caso en empresas de Ambato, Ecuador
}

\section{Corporate Social Responsibility in the inclusion of people with Disability. Case Study Ambato Companies, Ecuador} Fanny Paulina Pico Barrionuevo es Profesora e Investigadora en la carrera de Trabajo Social de la Universi-
dad Técnica de Ambato (Ecuador) (paulinapico@hotmail.com) (http://orcid.org/0000-0002-2276-8198)

Silvia Susana Torres es Profesora e Investigadora en la carrera de Trabajo Social de la Universidad Técnica de Ambato (Ecuador) (stcvita@yahoo.com) (http://orcid.org/0000-0002-6824-8518)

\begin{abstract}
Resumen
La inclusión de las Personas con Discapacidad (PCD) en el ámbito laboral aún tiene falencias que se debe ir corrigiendo, considerando que este grupo ha sentido la exclusión laboral de alguna u otra forma. El sector empresarial, protagonista principal del mercado laboral y actor fundamental en la realización de una verdadera inclusión, ya sea por cumplimiento de normativas legales establecidas o a su vez por realizar Responsabilidad Social Empresarial, tiene en sus manos la inclusión laboral de personas con esta condición. El objetivo del presente trabajo es identificar la realidad de las empresas en la inclusión laboral de las personas con discapacidad. Para el efecto se aplica un diseño metodológico de tipo cuali-cuantitativo en base a un marco teórico revisado para luego identificar valoraciones que permitan reunir evidencias y emitir un juicio de valor en la investigación. Se aplica el tipo de muestra probabilística aleatoria simple a través de la cual cada elemento de la población tiene la misma oportunidad de ser elegida. La investigación se realiza a través de una encuesta establecida de cuatro dimensiones: i) planificación; ii) inclusión; iii) equidad y; iv) desarrollo. De las 80 empresas encuestadas, el 11,70\% corresponden a aquellas que realizan las mejores prácticas empresariales. También destaca el sector comercial en los ejes de inclusión, equidad y desarrollo. El eje de desarrollo es el que tiene menor porcentaje, por lo que se le debe considerar con mayor interés para mejorar los procesos de inclusión de PcD.
\end{abstract}

\begin{abstract}
The inclusion of persons with disabilities (PcD), in the field of labor still has flaws that should be corrected, considering that this group has been the exclusion of some form, the business sector of the labor market, a key actor in the realization of a true inclusion, either by legal compliance or by making corporate social responsibility, the labor inclusion should be a fundamental part of each company. The aim of this work is to identify the reality of companies in the labor market inclusion of persons with disabilities, to the effect applies a methodology of qualitative-quantitative research on the basis of a revised theoretical framework to then identify ratings to gather evidence and make a judgment of value to the investigation, applies the type of simple random probability sample through which each element of the population has an equal chance of being chosen, the research is performed through a set of four dimensions: Planning, inclusion, equity and development. Of the 80 companies surveyed the $11.70 \%$ correspond to those that perform the best business practices, it also highlights the Commercial Sector in the areas of: inclusion, equity and development. The axis of development is the one that has a lower percentage should be considered to improve the processes that include it.
\end{abstract}

\section{Palabras clave | keywords}

Responsabilidad social, empresa privada, impedido, grupo desfavorecido, trabajo, incapacidad. Social responsibility, private enterprises, disabled persons, vulnerable groups, labour, disabilities. 


\section{Introducción y estado de la cuestión}

En la actualidad se han establecido mecanismos para realizar la inclusión de personas con discapacidad en el sector empresarial. Sin embargo existen varias razones por las cuales este vulnerable grupo no ha logrado tener una inserción total en cada una de las empresas. Por esta razón, la presente investigación pretende identificar la realidad de las empresas con respecto a la inclusión laboral de las personas con discapacidad. En el país se ha puesto en vigencia la Ley Orgánica de Discapacidades, que manifiesta en su artículo 47 sobre la obligatoriedad de contratación a personas con discapacidad:

Artículo 47.- Inclusión laboral.- La o el empleador público o privado que cuente con un número mínimo de veinticinco (25) trabajadores está obligado a contratar, un mínimo de cuatro por ciento (4\%) de personas con discapacidad, en labores permanentes que se consideren apropiadas en relación con sus conocimientos, condiciones físicas y aptitudes individuales, procurando los principios de equidad de género y diversidad de discapacidades. El porcentaje de inclusión laboral deberá ser distribuido equitativamente en las provincias del país, cuando se trate de empleadores nacionales; y a los cantones, cuando se trate de empleadores provinciales (Asamblea Nacional República del Ecuador, 2012).

En este contexto existe una obligatoriedad en la contratación de personas con discapacidad. Por otro lado, la Ley Orgánica de Discapacidad, establece en su artículo 49 la deducción del 150\% adicional para el cálculo de la base imponible del impuesto a la Renta otorgando un incentivo a la empresa privada en la contratación de las Personas con Discapacidad (PcD). Cabe indicar que existe cierto desconocimiento sobre este incentivo en la empresa privada, pues existe una desidia aparente ante la realidad de la inclusión de PcD.

\subsection{La responsabilidad social empresarial (RSE)}

Existen varias discusiones sobre las acciones que realiza la empresa en lo relativo con la responsabilidad social. Sin embargo se debe aclarar que cada individuo que trabaja en una organización tiene una ética y un sentido de responsabilidad, lo cual se proyecta en la organización (Banco Interamericano de Desarrollo, 2011).

En España se analizan a profundidad los temas de responsabilidad social empresarial en el ámbito de la inclusión de personas con discapa- 
cidad. En el caso de las personas con discapacidad intelectual, realizan la inclusión a través de la legislación vigente y conjuntamente con la práctica de Responsabilidad Social Empresarial, que tiene que ver con la integración de personas con discapacidad o la igualdad de las personas (Blanco Prieto, 2015).

El término discapacidad tiene varias definiciones. Una de ellas que se manifestó en la Convención de los Derechos de las Personas con Discapacidad 2006 (como se cita en Ministerio de Relaciones Laborales - CONADIS, 2013) establece que:

(la) discapacidad es un concepto que evoluciona y que resulta de la interacción entre las personas con deficiencias y las barreras debidas a la actitud y al entorno que evitan su participación plena y efectiva en la sociedad, en igualdad de condiciones con las demás.

En la provincia de Tungurahua existen 2158 personas con discapacidad laboralmente activas, de las cuales el 55,46\% tiene discapacidad física (Consejo Nacional de Discapacidades CONADIS - Instituto Ecuatoriano de Seguridad Social IESS, 2016).

\subsection{Discapacidad y la inclusión laboral}

Las personas con discapacidad tienen una menor participación en el mercado laboral, inclusive con menos opciones aquellas con una discapacidad significativa. En este sentido existen organismos e instituciones que de alguna manera forman un canal entre las personas con discapacidad y la empresa privada para lograr su inserción laboral. En España, por ejemplo, existen los Centros Especiales de Empleo (CEE), los cuales son la principal vía de integración de las personas con discapacidad. El objetivo principal de estos centros es reducir el desempleo y la inactividad de los trabajadores con discapacidad (Rodríguez \& Cueto, 2013).

En otras latitudes, en Paraguay las instituciones privadas tienen en su historial alguna forma de relación laboral con las personas con discapacidad, siendo como factores de contratación la responsabilidad social y la iniciativa de diferentes entes gubernamentales y no gubernamentales los que dan hincapié al trabajo como derecho (Reyes Giménez, 2015).

En el Ecuador sobre este tema de discapacidad en el ámbito laboral, se analizan dos aspectos que tienen que ver con la limitada inserción en el mercado laboral. Primero con el grupo vulnerable $(\mathrm{PcD})$ quienes 
tienen un bajo nivel educativo, debido a que existen barreras de comunicación, al medio físico y a la información, lo que impiden la formación y capacitación de este grupo vulnerable (Consejo Nacional de la Igualdad de Discapacidades CONADIS, 2013). Por otro lado se encuentra el sector empresarial, que tiene resistencia hacia la contratación de personas con discapacidad por el prolongado proceso de integración laboral considerando la adaptación del empleado, empleador y equipo de trabajo, que además influye en la falta de aplicación de medidas de acción afirmativa para procesos de selección de personal (adaptación de test y material de selección, evaluación de conocimientos, competencias, habilidades y destrezas). De acuerdo con los requerimientos de cada discapacidad y de alguna forma se presentan irregularidades ante el cumplimiento de la Ley Reformatoria al Código de Trabajo (Ley de cuotas que establece $4 \%$ ).

Continuando en el mismo contexto, en el Ecuador se ha establecido un marco legal para el empleador público o privado que favorece la inserción de las PcD. Así, en el artículo 42, numeral 33 del Código de Trabajo se determina que:

(...) el empleador público o privado, que cuente con un número mínimo de veinticinco trabajadores, está obligado a contratar, al menos, a una persona con discapacidad, en labores permanentes que se consideren apropiadas en relación con sus conocimientos, condición física y aptitudes individuales, observándose los principios de equidad de género y diversidad de discapacidad. A partir del año 2009, el porcentaje obligatorio de contratación de personas con discapacidad, es del 4\% del total de trabajadores de cada empresa o patrono persona natural.

\section{Fundamentos y propósito del estudio}

Sea que la empresa quiera realizar una aplicación de Responsabilidad Social Empresarial a través de buenas prácticas empresariales en la inclusión de personas con discapacidad, es importante identificar cómo el grupo de $\mathrm{PcD}$ ha ingresado en el ámbito laboral empresarial y cómo actúa el empleador. Considerando que en el Ecuador ya se han establecido normativas legales y mejores prácticas para la inclusión de las personas con discapacidad, de tal manera que se establezca una relación socio-laboral entre estos dos actores, en consecuencia se ve la necesidad de realizar una primera investigación diagnóstica-exploratoria verifi- 
cando las variables de estudio, a través de dimensiones que incluyen criterios como la equidad e inclusión que son elementos primordiales para la atención a la población con discapacidad. En este sentido, la investigación se basa en los siguientes paradigmas:

Tabla 1. Paradigmas sobre equidad e inclusión social en el mercado laboral

\begin{tabular}{|l|l|l|l|}
\hline Equidad & \multicolumn{1}{|c|}{ Liberal } & \multicolumn{1}{c|}{ Marxista } & \multicolumn{1}{c|}{ Posestructuralista } \\
\hline $\begin{array}{l}\text { Generación de equi- } \\
\text { paración o igualdad } \\
\text { de oportunidades }\end{array}$ & $\begin{array}{l}\text { Supera las con- } \\
\text { diciones sociales, } \\
\text { políticas y económi- } \\
\text { cas que producen la } \\
\text { dominación }\end{array}$ & $\begin{array}{l}\text { Reconocimiento y potencia- } \\
\text { ción de la multiculturalidad } \\
\text { (esta se fundamenta más en la } \\
\text { diferencia que en la oportu- } \\
\text { nidad) }\end{array}$ \\
\hline $\begin{array}{l}\text { Minorías como } \\
\text { objetos de una } \\
\text { política que provee } \\
\text { un tratamiento dife- } \\
\text { rencial, generalmente } \\
\text { focalizado }\end{array}$ & $\begin{array}{l}\text { Se reconocen las } \\
\text { diferencias culturales } \\
\text { de las minorías; éstas } \\
\text { se explican desde el } \\
\text { modelo de la des- } \\
\text { ventaja social. }\end{array}$ & $\begin{array}{l}\text { Minorías como sujetos partí- } \\
\text { cipes en la construcción de la } \\
\text { política }\end{array}$ \\
\hline $\begin{array}{l}\text { Diversidad orientada } \\
\text { a la asimilación y al } \\
\text { individualismo }\end{array}$ & $\begin{array}{l}\text { Diversidad orientada } \\
\text { a generar contra los } \\
\text { grupos hegemónicos } \\
\text { en lo social cultural y } \\
\text { político }\end{array}$ & $\begin{array}{l}\text { Diversidad orientada al diálogo } \\
\text { e integración intercultural, la } \\
\text { identificación y los consensos } \\
\text { disensos }\end{array}$ \\
\hline $\begin{array}{l}\text { Inclusión } \\
\text { Compensar las } \\
\text { desigualdades con } \\
\text { las que los alum- } \\
\text { nos acceden a la } \\
\text { escuela. Medidas in- } \\
\text { dividuales centradas } \\
\text { en propuestas de } \\
\text { adaptaciones curri- } \\
\text { culares específicas o } \\
\text { programas compen- } \\
\text { satorios }\end{array}$ & $\begin{array}{l}\text { Desarrollar proce- } \\
\text { sos educativos en } \\
\text { programas sociales y } \\
\text { económicos, donde } \\
\text { toda la comunidad } \\
\text { educativa luche por } \\
\text { reducir los orígenes } \\
\text { de desigualdad y la } \\
\text { exclusión, que ge- } \\
\text { neralmente se sitúa } \\
\text { fuera de la escuela }\end{array}$ & $\begin{array}{l}\text { Es una actitud un sistema de } \\
\text { valores y creencias, no una ac- } \\
\text { conjunto de acciones. Implica } \\
\text { reconocer unos ejercicios de } \\
\text { poder, unos lenguajes, unas } \\
\text { relaciones por descontruir y } \\
\text { reorientar. Exige ofrecer una } \\
\text { propuesta de actuación que } \\
\text { impida la transformación de } \\
\text { las diferencias en símbolo de } \\
\text { marginación. }\end{array}$ \\
\hline
\end{tabular}

Fuente: Tomado de Díaz, O.C (1) Equidad, inclusión y discapacidad. CD Memorias II Foro Distrital de Discapacidad, Bogotá, 2005. Citado en (García Ruiz \& Fernández Moreno, 2005)

Las respuestas de inclusión social, requieren de unas propuestas políticas en las cuales se les reconozcan a todos los sujetos como protagonistas y dependerá del interés del sujeto en cada uno de los escenarios, como puede ser educativo o laboral (García-Ruiz \& Fernández-Moreno, 2005). 
En el momento de realizar la inducción, ésta debe ser 100\% inclusiva y adecuada a las necesidades específicas de las personas con discapacidad. En el caso de que se vea en la necesidad de tener un intérprete de lengua de señas -por ejemplo- será imprescindible realizarlo de esta forma (Ministerio de Relaciones Laborales - Dirección de Atención a Grupos Prioritarios - Conadis, 2013).

En el Ecuador, el Ministerio de Inclusión Económica y Social, según el Acuerdo Ministerial No 000154, en su artículo 3 establece la gestión de los principios de igualdad, inclusión, equidad, universalidad e integralidad (Ministerio de Inclusión Económica y Social, 2013), mismos que se consideran en la aplicación de diferentes instrumentos de valoración para la aplicación de procesos en el ámbito laboral y que tienen que ver con las $\mathrm{PcD}$.

\section{Material y métodos}

El objetivo general del presente trabajo es identificar la realidad de las empresas en la inclusión laboral de las personas con discapacidad. Para la realización de la investigación se aplicó el método cuantitativo, el cual se fundamenta en la medición de las características de fenómenos sociales que derivaron de un marco conceptual adecuado a la problemática estudiada (Bernal, 2010). Considerando los criterios establecidos en el cuestionario se determinarán valores que permitirán emitir juicios de valor de acuerdo a los resultados presentados.

También se aplicará el método cualitativo o método no tradicional, en donde se pretende entender los diferentes tipos de situaciones sociales como un todo, considerando sus propiedades y su dinámica (Bernal, 2010).

El grupo analizado será el sector empresarial y las personas con discapacidad en la ciudad de Ambato (Tungurahua, Ecuador). Se aplicó una muestra probabilística aleatoria simple a través de la cual cada elemento de la población tiene la misma oportunidad de ser elegida, y donde la probabilidad de seleccionar a cualquier miembro en particular de la población la primera vez es $1 / \mathrm{N}$ (Berenson, Levine, \& Krehbiel, 2001), de tal forma que $\mathrm{N}=160$ y $\mathrm{n}=80$. El listado de empresas fue proporcionado por la Fundación Cuesta Holguín.

Las empresas participantes se desarrollan en el sector privado y están involucradas en diferentes actividades sectoriales. Se aplicó una encuesta a 80 de ellas, tal como se extrae en la tabla 2: 
Tabla 2. Empresas participantes por Sector

\begin{tabular}{|l|c|}
\hline \multicolumn{1}{|c|}{ Actividad Sectorial } & $\%$ \\
\hline Sector Industrial & 28,57 \\
\hline Sector Comercial & 25,97 \\
\hline Sector Servicios & 45,45 \\
\hline
\end{tabular}

Para la realización del trabajo se aplicó una encuesta establecida a través de cuatro dimensiones: i) planificación; ii) inclusión; iii) equidad y; iv) desarrollo. Cada eje consta de ítems detallados de la siguiente manera:

- Planificación: Incluye 4 ítems y valora si la empresa cuenta con una planificación inicial en el proceso de selección. Establece si se cumple con el porcentaje establecido en la normativa que incluye el tipo de discapacidad, la realización del proceso de selección y la inducción de las PcD.

- Inclusión: Incluye 4 ítems y revisa la inclusión social en la actividad laboral, la accesibilidad física dependiendo del tipo de discapacidad, así como la señalización e información y comunicación adecuada a las PcD.

- Equidad: Incluye 4 ítems y analiza la equidad de derechos y beneficios, sensibilización, la reubicación laboral y el reconocimiento al buen desempeño laboral.

- Desarrollo: Incluye 4 ítems y establece la realización de capacitación, evaluación, desarrollo y promoción así como también la adaptación del puesto de trabajo.

Cada ítem fue valorado a través de tres criterios: i) mejor práctica; ii) buena práctica y; iii) en proceso, entendiéndose cada una de ellas de la siguiente manera:

- Mejor Práctica: Va más allá del cumplimiento de la normativa (Ley Orgánica de Discapacidades, revisada ut supra), es decir que no solamente cumple con la norma sino que además desarrolla mejoras en cada proceso (excelencia), y se lo valora con una puntuación de 10.

- Buena Práctica: Cumple con la normativa establecida y se valora con una puntuación de 5

- En Proceso: Tiene planificado realizarlo y se le valora con una puntuación de 0 


\section{Análisis y resultados}

Se han identificado los resultados luego de haber aplicado las encuestas. Se determinan porcentajes y aplicando la media aritmética se evidencian resultados agrupados por dimensiones, como se detalla en la tabla 3:

\section{Tabla 3. Aplicación de cada dimensión en las empresas por actividad sectorial}

\begin{tabular}{|l|c|c|c|c|}
\hline \multicolumn{1}{|c|}{$\begin{array}{c}\text { Actividad Sectorial } \\
\text { Empresarial }\end{array}$} & Planificación & Inclusión & Equidad & Desarrollo \\
\hline Sector Industrial & $9,09 \%$ & $11,08 \%$ & $8,81 \%$ & $6,96 \%$ \\
\hline Sector Comercial & $9,38 \%$ & $12,03 \%$ & $10,78 \%$ & $8,75 \%$ \\
\hline Sector Servicios & $9,87 \%$ & $10,08 \%$ & $7,41 \%$ & $6,79 \%$ \\
\hline
\end{tabular}

El sector comercial tiene porcentajes que destacan del resto en cuanto a las variables inclusión, equidad y desarrollo, mientras que en el sector servicios destaca en la planificación. La tabla 4 muestra los resultados sobre las mejores prácticas empresariales por actividad sectorial:

Tabla 4. Mejores prácticas empresariales

\begin{tabular}{|l|c|c|c|}
\hline \multicolumn{1}{|c|}{ Actividad Sectorial } & Mejor Práctica & Buena Práctica & En Proceso \\
\hline Sector Industrial & $16,27 \%$ & $51 \%$ & $34,09 \%$ \\
\hline Sector Comercial & $13,95 \%$ & $56,85 \%$ & $29,2 \%$ \\
\hline Sector Servicios & $8,57 \%$ & $60,89 \%$ & $30,31 \%$ \\
\hline
\end{tabular}

Se identifican como mejores prácticas empresariales al sector industrial, con un resultado de $16,27 \%$, en segundo lugar el sector comercial con un $13,95 \%$ y en tercer lugar el sector servicios con un $8,57 \%$. De esta manera se establece un cuadro consolidado (gráfico 1) con la información sobre las mejores prácticas empresariales en la Inclusión de personas con discapacidad.

De las 80 empresas encuestadas, el 11,70\% corresponden a aquellas que realizan las mejores prácticas empresariales, un 57,5\% realizan buenas prácticas empresariales, mientras que un $30,9 \%$ se encuentran en proceso. 


\section{Gráfico 1. Cuadro consolidado de interrelación entre mejores y buenas prácticas y empresas "en proceso"}

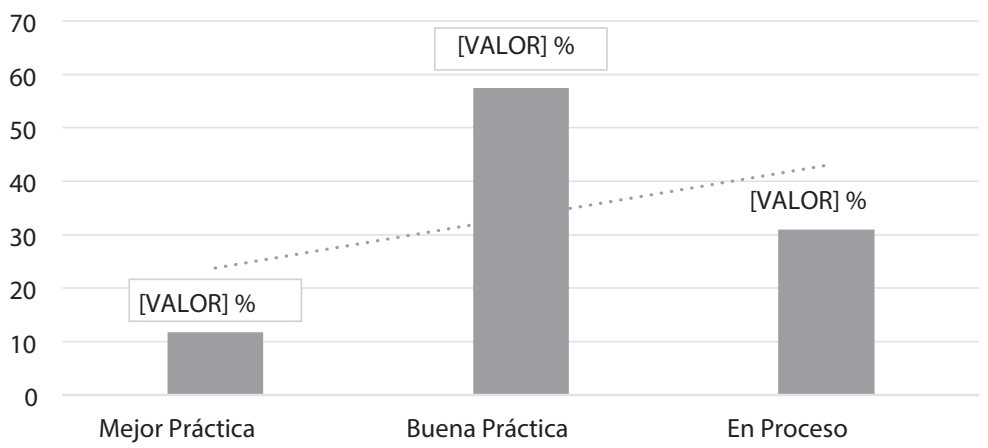

\section{Discusión y conclusiones}

De los cuatro ejes establecidos para la identificación de las mejores prácticas se analiza primeramente la «planificación», la cual se observa un alto porcentaje en el sector de servicios con un 9,87\%, lo cual motiva a las empresas a tener un acercamiento de las empresas hacia organismos como el Consejo Nacional de Discapacidades (CONADIS) y a su vez con los Servicios de Integración Laboral (SILL), organismos que están encargados de brindar asesoramiento empresarial, así como también charlas de sensibilización en materia de inclusión.

También se analiza que los procesos de selección y de inducción deben ser adaptados a las personas con discapacidad, considerando que la empresa debe construir herramientas inclusivas (técnicas de comunicación) que les permitan socializar el código de ética interno, el manual administrativo, el comité de seguridad y salud ocupacional o cualquier otra información relevante con relación a horarios y sueldos (Ministerio de Relaciones Laborales - Dirección de Atención a Grupos PrioritariosConadis, 2013).

El eje de la «inclusión» analiza las actividades sociales y culturales que realiza la empresa y cómo es la participación de las Personas con Discapacidad (PcD). También incluye accesibilidad física, señalización y comunicación en el sector comercial, apreciándose un valor alto con respecto a los otros sectores con un $12,03 \%$. En este sentido, se debe 
indicar que las personas con discapacidad deben tener el mejor trato el cual debe ser prioritario y no diferente, caracterizado por el respeto (Ministerio de Relaciones Laborales - Dirección de Atención a Grupos Prioritarios - Conadis, 2013).

El eje de la «equidad» analiza los beneficios y derechos que tienen las $\mathrm{PcD}$ y si los colaboradores han sido sensibilizados frente a la discapacidad. También si la empresa cuenta con procesos de reubicación socio-laboral y si la organización tiene políticas de reconocimiento por el buen desempeño laboral. En este ámbito el sector comercial cuenta con un 10,78\% sobre los sectores industrial y de servicios.

Finalmente el eje de «desarrollo», que es uno de los ejes que se deben mejorar pues es el más bajo con respecto a los demás, analiza cómo la empresa incluye a las $\mathrm{PcD}$ en procesos de capacitación y formación, así como también analiza si el sistema de evaluación es acorde a los requerimientos de las $\mathrm{PcD}$, ya que según sean las habilidades o destrezas de las personas con discapacidad, se deberán adaptar los instrumentos o realizar ajustes a los mismos (Ministerio de Relaciones Laborales Dirección de Atención a Grupos Prioritarios - Conadis, 2013).

Lo anterior incluye además una revisión sobre las promociones y desarrollo en el ámbito laboral. Algo importante que recalcar es la necesidad de realizar adaptaciones del puesto de trabajo en caso de que sea necesario, de acuerdo al tipo de discapacidad que tenga el colaborador, al igual que en las anteriores dimensiones, los ejes de «inclusión» y «equidad» en el sector comercial destacan un 8,75\% más que los demás sectores.

Finalmente, de las 80 empresas encuestadas el 11,70\% corresponden a aquellas que realizan las mejores prácticas empresariales; quiere decir que sí existe un grupo empresarial preocupado por alcanzar el bienestar y la inclusión de su PcD. Por su parte, un grupo correspondiente al $57,5 \%$-es decir, más de la mitad de empresas encuestadas- obtienen una evaluación positiva de realizar buenas prácticas empresariales, mientras que un 30,9\% se encuentran en proceso de aplicación.

Con respecto al objetivo señalado se puede indicar que las empresas tienen un papel importante en el ámbito laboral y en tal virtud se evidencia que más de la mitad de empresas encuestadas realizan buenas prácticas empresariales, estableciendo procesos de inclusión de acuerdo a los requerimientos de las Personas con Discapacidad (PcD). Por otro lado, se deja abierta la posibilidad de investigaciones futuras para ana- 
lizar cada una de las dimensiones establecidas, así como también investigar sobre las necesidades de los sectores empresariales en la adecuada ubicación de las Personas con Discapacidad.

Las limitaciones del estudio fueron básicamente de tiempo, debido a que se ha determinado como un diagnóstico inicial de cómo se encuentra el sector empresarial frente a la inclusión de personas con discapacidad. En este sentido es importante establecer que se debe estudiar el comportamiento de las variables analizadas en el lapso de un año para identificar nuevos resultados.

\section{Referencias}

Asamblea Nacional República del Ecuador. (25 de septiembre de 2012). Registro Oficial. Ley Orgánica de Discapacidades. Quito, Pichincha, Ecuador: Tribunal Constitucional de la República del Ecuador.

Banco Interamericano de Desarrollo (Mayo de 2011). Responsabilidad de la empresa ante la sociedad - Antonio Vives. La Responsabilidad Social de la empresa en América Latina. New York, Estados Unidos: BID.

Berenson, M. L., Levine, D. M., \& Krehbiel, T. C. (2001). Estadística para administración. México: Pearson Educación.

Bernal, C. A. (2010). Metodología de la Investigación. Bogotá: Pearson Educación.

Blanco Prieto, A. (2015). Responsabilidad social empresarial e integración laboral de personas con discapacidad intelectual. Estudio de Caso. Revista Española de Discapacidad, 211-217.

Consejo Nacional de Discapacidades CONADIS - Instituto Ecuatoriano de Seguridad Social IESS. (1 de julio de 2016). Estadísticas Conadis. Obtenido de Estadísticas Conadis: http://www.consejodiscapacidades.gob.ec/estadistica/index.html

Consejo Nacional de la Igualdad de Discapacidades CONADIS. (2 de diciembre de 2013). Agenda Nacional para la Igualdad en Discapacidades 2013 - 2017. Cambiando Vidas. Quito, Pichincha, Ecuador: Equipo Técnico Consejo Nacional de Discapacidades. Obtenido de http://www.planificacion.gob.ec/wp-content/uploads/downloads/2014/09/Agenda-Nacional-para-Discapacidades.pdf

Consejo Nacional para la Igualdad de Discapacidades. (1 de julio de 2016). Consejo Nacional para la Igualdad de Discapacidades. Obtenido de www.consejodiscapacidades.gob.ec/estadistica/index.html

Fundación General Ecuatoriana (2010). I Reconocimiento empresarial a las mejores prácticas en inclusión sociolaboral de personas con discapacidad. Quito: Fundación General Ecuatoriana.

García Ruiz, A. S., \& Fernández Moreno, A. (2005). La inclusión para las personas con discapacidad: entre la iguladad y la diferencia. Revista Ciencias de la Salud, 235246. Obtenido de http://www.redalyc.org/pdf/562/56230213.pdf

Ministerio de Inclusión Económica y Social. (9 de enero de 2013). Ministerio de Inclusión Económica y Social. Obtenido de http://www.inclusion.gob.ec/ 
wp-content/uploads/downloads/2013/02/ESTATUTO-ORGA\%CC\%81NICO-POR-PROCESOS-MIES-al-09-enero-2013-5.pdf

Ministerio de Relaciones Laborales - CONADIS. (4 de Diciembre de 2013). Ministerio de Trabajo. Manual de buenas prácticas para la Inclusión laboral de personas con discapacidad. Quito, Pichincha, Ecuador. Obtenido de Manual de Buenas Prácticas para la inclusión laboral de personas con discapacidad: http://www. trabajo.gob.ec/wp-content/uploads/2013/12/MANUALFIN.pdf

Ministerio de Relaciones Laborales - Dirección de Atención a Grupos Prioritarios Conadis. (2013). Consejo de Discapacidades. Obtenido de Manual de Buenas prácticas para la Inclusión laboral de Personas con Discapacidad: http://www. consejodiscapacidades.gob.ec/wp-content/uploads/downloads/2014/06/manual_buenas_practicas_inclusion_laboral.pdf

Reyes Giménez, A. (2015). Empleabilidad de personas con discapacidad desde el marco rector de SENADIS, Paraguay. Revista Internacional de Integración en Ciencias Sociales, 209-222. Obtenido de http://revistacientifica.uaa.edu.py/index. $\mathrm{php} / \mathrm{riics} /$ article/view/268

Rodríguez, V., \& Cueto, B. (2013). El trabajo de las personas con discapacidad ante la crisis. Revista Internacional de Organizaciones, 61-86. Obtenido de https:// dialnet.unirioja.es/servlet/articulo?codigo $=4994810$ 